\title{
Microscopic Validation of Macroscopic In Vivo Images Enabled by Same-Slide Optical and Nuclear Fusion
}

\author{
Kazumasa Inoue ${ }^{1,2}$, Summer L. Gibbs ${ }^{1}$, Fangbing Liu ${ }^{1}$, Jeong Heon Lee ${ }^{1}$, Yang Xie ${ }^{1}$, Yoshitomo Ashitate ${ }^{1}$, \\ Hirofumi Fujii ${ }^{3}$, John V. Frangioni ${ }^{1,4,5}$, and Hak Soo Choi ${ }^{1}$ \\ ${ }^{I}$ Division of Hematology/Oncology, Department of Medicine, Beth Israel Deaconess Medical Center and Harvard Medical School, \\ Boston, Massachusetts; ${ }^{2}$ Department of Radiological Sciences, Tokyo Metropolitan University, Tokyo, Japan; ${ }^{3}$ Functional Imaging \\ Division, Research Center for Innovative Oncology, National Cancer Center Hospital East, Chiba, Japan; ${ }^{4}$ Department of Radiology, \\ Beth Israel Deaconess Medical Center, Boston, Massachusetts; and ${ }^{5}$ Curadel, LLC, Worcester, Massachusetts
}

It is currently difficult to determine the molecular and cellular basis for radioscintigraphic signals obtained during macroscopic in vivo imaging. The field is in need of technology that helps bridge the macroscopic and microscopic regimes. To solve this problem, we developed a fiducial marker (FM) simultaneously compatible with 2-color near-infrared (NIR) fluorescence (700 and $800 \mathrm{~nm}$ ), autoradiography, and conventional hematoxylin-eosin (HE) histology. Methods: The FM was constructed from an optimized concentration of commercially available human serum albumin, 700- and $800-\mathrm{nm}$ NIR fluorophores, 99mTc-pertechnetate, dimethyl sulfoxide, and glutaraldehyde. Lymphangioleiomyomatosis cells coexpressing the sodium iodide symporter and green fluorescent protein were labeled with $700-\mathrm{nm}$ fluorophore and ${ }^{99 \mathrm{~m} T c-p e r t e c h n a t a t e}$ and then administered intratracheally into CD-1 mice. After in vivo SPECT imaging and ex vivo SPECT and NIR fluorescence imaging of the lungs, 30- $\mu \mathrm{m}$ frozen sections were prepared and processed for $800-\mathrm{nm}$ NIR fluorophore costaining, autoradiography, and HE staining on the same slide using the FMs to coregister all datasets. Results: Optimized FMs, composed of $100 \mu \mathrm{M}$ unlabeled human serum albumin, $1 \mu \mathrm{M}$ NIR fluorescent human serum albumin, $15 \%$ dimethyl sulfoxide, and $3 \%$ glutaraldehyde in phosphate-buffered saline $(\mathrm{pH} 7.4)$, were prepared within $15 \mathrm{~min}$, displayed homogeneity and stability, and were visible by all imaging modalities, including $\mathrm{HE}$ staining. Using these FMs, tissue displaying high signal by SPECT could be dissected and analyzed on the same slide and at the microscopic level for 700-nm NIR fluorescence, 800-nm NIR fluorescence, autoradiography, and HE histopathologic staining. Conclusion: When multimodal FMs are combined with a new technique for simultaneous same-slide NIR fluorescence imaging, autoradiography, and HE staining, macroscopic in vivo images can now be studied unambiguously at the microscopic level.

Key Words: molecular and cellular basis of signal; multimodality imaging; fiducial marker; autoradiography; NIR fluorescence; HE staining

J Nucl Med 2014; 55:1899-1904

DOI: $10.2967 /$ jnumed.114.141606

\footnotetext{
Received Apr. 14, 2014; revision accepted Sep. 5, 2014.

For correspondence or reprints contact: Hak Soo Choi, Beth Israel Deaconess Medical Center, Room SL-436A, 330 Brookline Ave., Boston, MA 02215

E-mail: hchoi@bidmc.harvard.edu

Published online Oct. 16, 2014.

COPYRIGHT (C) 2014 by the Society of Nuclear Medicine and Molecular Imaging, Inc.
}

$\mathbf{T}$ he development of new clinical radiotracers for SPECT and PET is of critical importance $(1,2)$. However, validating that the signal obtained during in vivo radioscintigraphic imaging is emanating from the target of interest can be a challenge (3-5). Particularly difficult is the validation of signals at the cellular or molecular level.

Clinically, histopathologic staining with hematoxylin-eosin (HE) is the gold standard for identification of specific cells and tissues (6); however, ambiguity is common. To ensure proper identification, one or more immunomarkers are typically used, and precipitating pigments are used in conjunction with HE to label cells of interest $(3,7)$. The problem with conventional immunohistochemistry, however, is that the HE image itself is masked by the precipitating pigments (8). To solve this problem, our group has previously developed a technology to perform 2 independent wavelengths of near-infrared (NIR) fluorescence immunostaining on the same specimen and the same slide as conventional HE (Gibbs et al., unpublished data, September 2014). Thus, the unadulterated HE can be used in conjunction with immunomarkers to unambiguously identify cells or molecules of interest.

What is needed in the field of nuclear medicine is the ability to correlate radioscintigraphic signals measured using macroscopic in vivo imaging techniques with validation performed at the single-cell level. But few such techniques exist. For this reason, we focused the present study on developing an optimized fiducial marker (FM) that permits acquisition and coregistration of HE, NIR fluorescence, and autoradiography datasets on the same specimen and on same slide. We hypothesized that such a technology would enable unambiguous validation of macroscopic nuclear images at the microscopic level.

\section{MATERIALS AND METHODS}

\section{Preparation of HSA700 and HSA800}

Cy5.5 N-hydroxysuccinimide (NHS) ester was purchased from GE Healthcare, and ZW800-1 was synthesized as previously reported $(9,10)$. They were diluted to $10 \mathrm{mM}$ in dimethyl sulfoxide and stored at $-80^{\circ} \mathrm{C}$ under reduced-light conditions. Human serum albumin (HSA) and glutaraldehyde were purchased from Sigma and Electron Microscopy Sciences, respectively. ${ }^{99 \mathrm{~m}} \mathrm{Tc}$-pertechnatate was purchased from Cardinal Health. The red blood cell labeling kit (UltraTag) was purchased from Covidien. Rabbit polyclonal anti-green fluorescent protein (GFP) antibody was purchased from Abcam. Magnesium chloride and trypsinVersene were purchased from Fisher Scientific and Cambrex, respectively. HSA suspended in phosphate-buffered saline (PBS), pH 7.8, was first purified using gel filtration chromatography with a Bio-Gel P-6 desalting 


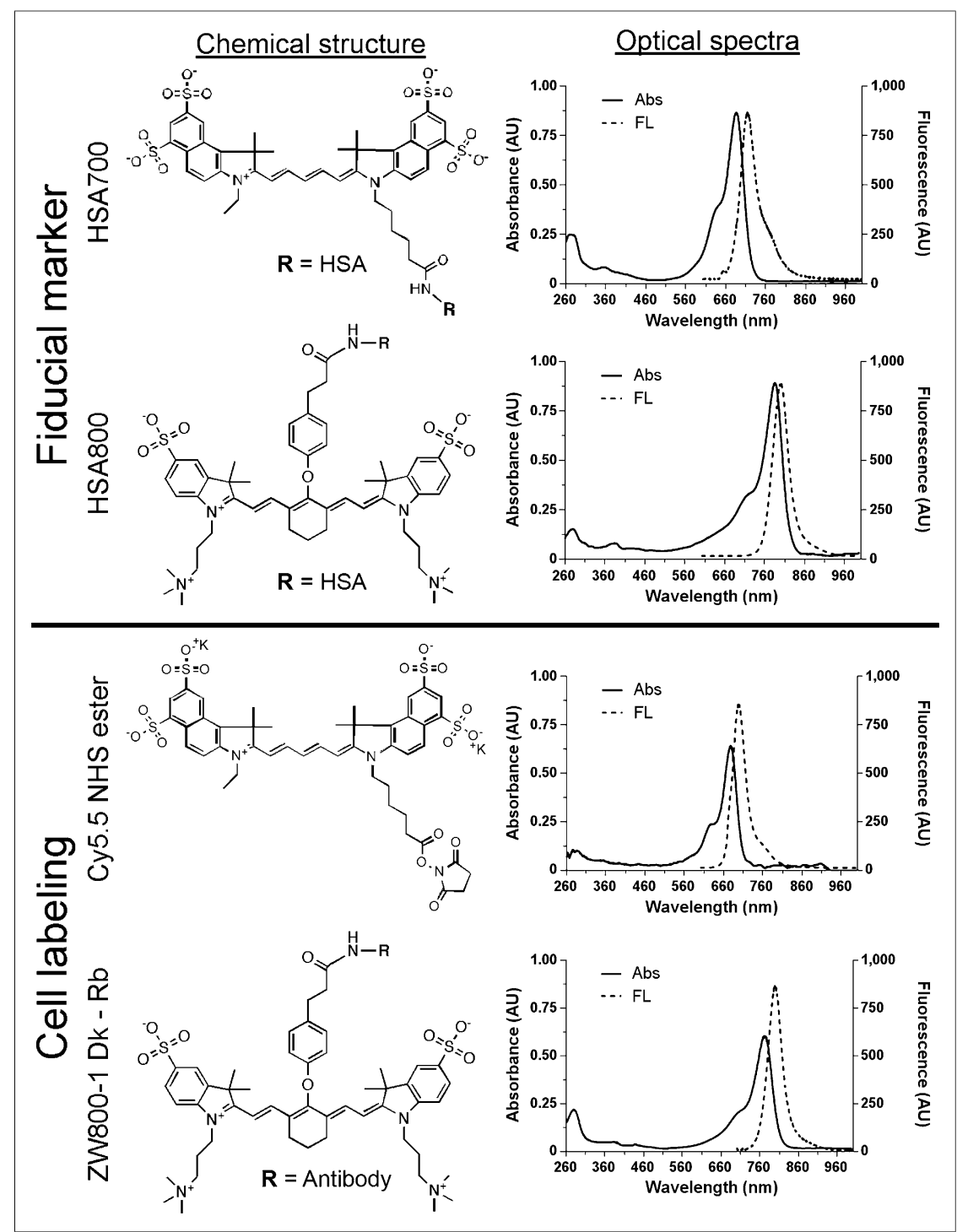

FIGURE 1. Chemical structure and optical spectra of FMs and cell-labeling reagents. HSA700 and HSA800 for FMs were prepared by conjugating Cy5.5 and ZW800-1, respectively, to purified HSA. Cells were labeled using Cy5.5 NHS ester (700-nm fluorophore) and ZW800-1-conjugated donkey a antirabbit antibody. Shown are chemical structures (left) and optical spectra (right). Abs = absorbance; $\mathrm{AU}=$ arbitrary unit; $\mathrm{FL}=$ fluorescence.

column to remove small molecules that interfere with covalent conjugation by NHS esters. Two equivalents of Cy5.5 NHS ester or ZW800-1 NHS ester were added to the purified HSA in PBS, pH 7.8, and incubated for $3 \mathrm{~h}$ to form a stable amide linkage. HSA700 and HSA800 were purified using gel filtration chromatography as above, and the labeling ratio was calculated from the ratio of extinction coefficients between the HSA $\left(\varepsilon_{280 \mathrm{~nm}}=32,900 \mathrm{M}^{-1} \mathrm{~cm}^{-1}\right)$ and the NIR fluorophore (Cy5.5, $\varepsilon_{700 \mathrm{~nm}}=250,000 \mathrm{M}^{-1} \mathrm{~cm}^{-1} ; \mathrm{ZW} 800-1, \varepsilon_{770 \mathrm{~nm}}=249,000 \mathrm{M}^{-1} \mathrm{~cm}^{-1}$ ). The final NIR fluorophore labeling ratios for HSA700 and HSA800 were 0.95 and 0.96 , respectively. All steps were performed under reduced-light conditions. The optical spectra of HSA700 and HSA800 were obtained with an ultraviolet-visible and fluorescence spectrophotometer (Varian). Final products were stored as $0.2 \mathrm{mM}$ stock solutions in PBS, $\mathrm{pH} 7.4$, at $4{ }^{\circ} \mathrm{C}$.

\section{Optimization of the Multimodality Fiducial Marker}

Unlabeled HSA, HSA700, HSA800, ${ }^{99 m}$ Tc-pertechnatate, dimethyl sulfoxide, and glutaraldehyde concentrations were varied systematically, and the homogeneity and stability of conjugation to an amine-coated
Superfrost Plus (VWR) microscope slide were measured. Reagents were gently stirred together in a vortex mixer at room temperature (RT), and $0.2 \mu \mathrm{L}$ of the solution was spotted by hand onto the glass slide, which contained tissue or did not, depending on the experiment. The FM was then incubated for $15 \mathrm{~min}$ at RT, washed with distilled water, and dried for 15 min at RT. Tissue samples were circled with a liquid blocker (PAP pen; Sigma) to protect from washing procedures. The glass slide was exposed to a phosphor imaging screen overnight at $-20^{\circ} \mathrm{C}$. In the following step, the slide was moved to RT and the protective film placed on the FM or the tissue sample removed gently to reduce potential tissue damage. The phosphor imaging screen was read using a Typhoon 9400 system (GE Healthcare). The pixel size and depth were set to $25 \mu \mathrm{m}$ and 16 bits, respectively. The phosphor imaging screen was erased for a minimum of $2 \mathrm{~h}$ before the next experiment and was confirmed to be back to baseline. HSA700 and HSA800 were imaged on a TE 300 microscope system (Nikon) equipped with mercury and xenon excitation sources (Chiu Technical Corp.) and an Orca-ER 12-bit camera (Hamamatsu). To obtain fluorescence images of HSA700, the xenon light source was passed through a $650 / 45-n m$ band-pass excitation filter and a 700/35-nm band-pass emission filter (Chroma Technology). To obtain fluorescence images of HSA800, the xenon light source was passed through a 750/50-nm band-pass excitation filter and an 810/40-nm band-pass emission filter (Chroma Technology). Slides were then HE-stained using a standard clinical protocol. The signal intensity, homogeneity, and shape of all obtained fluorescence images were compared before and after HE staining.

\section{Labeling and Imaging of Lymphangioleiomyomatosis cells}

Lymphangioleiomyomatosis 621-327 cells (11) were cultured in a 50/50 mixture of Dulbecco modified Eagle medium/F12 (Invitrogen) supplemented with epidermal growth factor $(10 \mathrm{ng} / \mathrm{mL}$; SigmaAldrich), hydrocortisone (200 nM; Sigma-Aldrich), insulin $(25 \mu \mathrm{g} / \mathrm{mL}$; Sigma-Aldrich), sodium selenite (50 nM; Sigma-Aldrich), transferrin (10 $\mu \mathrm{g} / \mathrm{mL}$; Sigma-Aldrich), ferrous sulfate (1.6 $\mu \mathrm{M}$; Sigma-Aldrich), and fetal bovine serum (15\%, Gemini). The cells were grown at approximately $70 \%$ confluence on a 10 -cm-diameter tissue culture plate and washed once with PBS containing $1 \mathrm{mM} \mathrm{MgCl} 2$. The cells were labeled with $2 \mu \mathrm{M}$ Cy5.5 NHS ester in $10 \mathrm{~mL}$ of $\mathrm{PBS} / \mathrm{MgCl}_{2}$ for $30 \mathrm{~min}$ at $37^{\circ} \mathrm{C}$ and washed 3 times, and $10 \%$ of the components of the UltraTag red blood cell labeling kit were added. Dose escalation studies confirmed that $2 \mu \mathrm{M}$ or lower of NHS ester treatment of the cell surface had no impact on cell viability for up to $24 \mathrm{~h}$. The cells were mixed by gently inverting the components of the red blood cell kit for $10 \mathrm{~min}$ at $37^{\circ} \mathrm{C}$, and then $37 \mathrm{MBq}(1 \mathrm{mCi})$ of ${ }^{99 \mathrm{~m}} \mathrm{Tc}$-pertechnetate were added to the plate. After gentle rocking for $2 \mathrm{~h}$ at $37^{\circ} \mathrm{C}$, the cells were washed once with buffer and trypsinized by adding $2 \mathrm{~mL}$ of trypsin-Versene (Sigma-Aldrich). The cells labeled with Cy5.5 and ${ }^{99 \mathrm{~m} T c}$ were then washed 3 times by centrifuging at 2,000 rpm for $5 \mathrm{~min}$. The supernatant was discarded carefully 


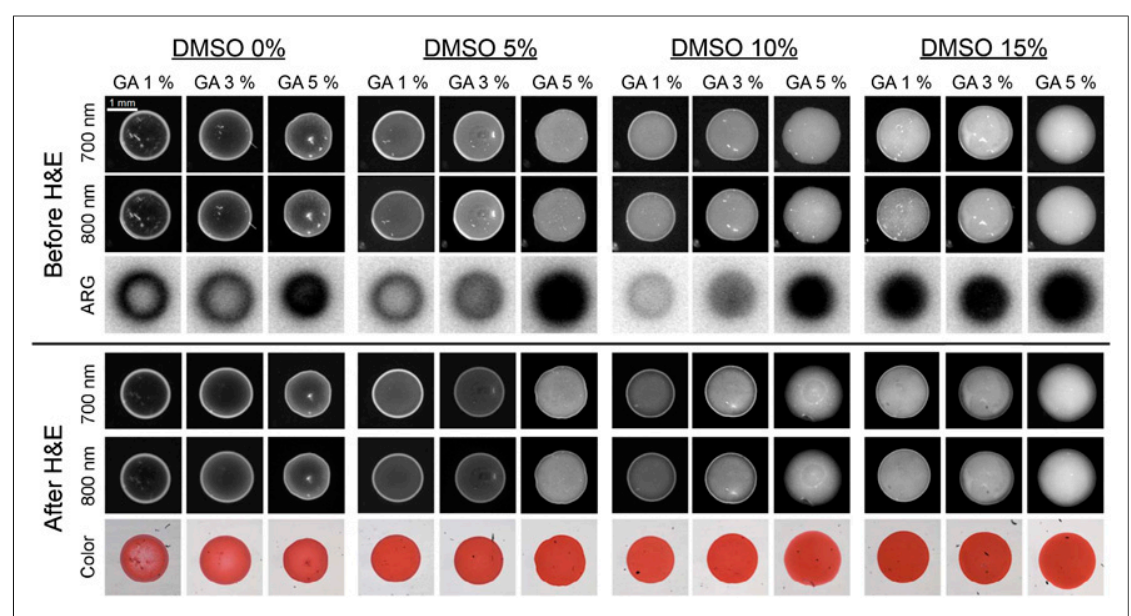

FIGURE 2. Optimization of FMs. Unlabeled HSA $(100 \mu \mathrm{M}), \operatorname{HSA} 700(1 \mu \mathrm{M}), \operatorname{HSA} 800(1 \mu \mathrm{M})$, and 99mTc-pertechnetate $(0.37 \mathrm{kBq}[0.01 \mu \mathrm{Ci}] / \mu \mathrm{L})$ in PBS, pH 7.4, were fixed while concentrations of glutaraldehyde and dimethyl sulfoxide were systematically varied as shown. NIR fluorescence signal intensity was measured before (top) and after (bottom) HE staining. Autoradiography was measured before HE staining only. All NIR fluorescence images have identical exposure times and normalizations. Data are representative of 3 independent experiments. ARG = autoradiography; DMSO = dimethyl sulfoxide; GA = glutaraldehyde.

so as not to disturb the cell pellet. The radiolabeling efficiency was over $80 \%$. The cells were resuspended in $200 \mu \mathrm{L}$ of $\mathrm{PBS} / \mathrm{MgCl}_{2}$ and loaded on the microscope glass slide, which was fixed with $2 \%$ formaldehyde for autoradiography scanning. Autoradiography was performed using the same parameters as described above. The signal of GFP was then imaged using a fluorescence microscope equipped with a 480/40-nm band-pass excitation filter and a 535/50-nm band-pass emission filter.

\section{Immunofluorescence Staining}

ZW800-1-conjugated donkey antirabbit secondary antibody was prepared as follows: $400 \mu \mathrm{L}$ of donkey antibody (stock concentration, $1.2 \mathrm{mg} / \mathrm{mL}$ ) was buffer-exchanged with PBS using spin columns (Vivaspin 500, 10-kDa molecular weight cutoff; GE Healthcare) to eliminate any sodium azide that might be present in the stock solution. The buffer exchange was performed using centrifugation at $8,000 \mathrm{rpm} 5$ times, 3 min each. The antibody solution was adjusted to $\mathrm{pH} 8$, and 5-25 equivalents of ZW800-1 NHS ester were added. After a 3-h reaction at RT, the NIR-antibody conjugate was purified using gel filtration chromatography as described above. Purified fractions were collected and concentrated to a $50-\mu \mathrm{L}$ volume by centrifugation at $10,000 \mathrm{rpm}$ for $3 \mathrm{~min}$. The labeling ratio, calculated from the ratio of extinction coefficients for the antibody $\left(\varepsilon_{280 \mathrm{~nm}}=150,000 \mathrm{M}^{-1} \mathrm{~cm}^{-1}\right)$ and ZW800-1 $\left(\varepsilon_{770 \mathrm{~nm}}=249,000 \mathrm{M}^{-1} \mathrm{~cm}^{-1}\right)$, was 1.5 . PBS containing $2.5 \%$ goat serum was added to the slide and incubated for $1 \mathrm{~h}$ at RT to block nonspecific protein binding sites. Then, the antirabbit polyclonal antiGFP antibody ( $1: 100$ dilution in PBS $+0.1 \%$ bovine serum albumin + $0.1 \%$ polysorbate 20 ) was added and incubated overnight at RT. After washing 3 times with PBS containing $0.5 \%$ polysorbate 20 (PBS/T), $5 \mu \mathrm{M}$ ZW800-1 conjugated secondary antibody was added and incubated for $2 \mathrm{~h}$ at RT. After incubation, the lymphangioleiomyomatosis cells were washed with PBS/T 3 times, 5 min each time. Lymphangioleiomyomatosis cells were then fixed in $2 \%$ paraformaldehyde in PBS for $15 \mathrm{~min}$ at RT and then stained with HE. After HE staining, color imaging and 800-nm anti-GFP immunofluorescence imaging were performed.

\section{In Vivo and Ex Vivo Imaging}

Animals used in this study were housed in a facility certified by the Association for Assessment and Accreditation of Laboratory Animal
Care and staffed by full-time veterinarians. Animal studies were performed under the supervision of the institutional animal care and utilization committee of Beth Israel Deaconess Medical Center in accordance with approved institutional protocol 155-2008. CD-1 mice of either sex, 8-10 wk old, were purchased from Charles River Laboratories. The mice were anesthetized intraperitoneally with ketamine $(100 \mathrm{mg} / \mathrm{kg})$ and xylazine $(10 \mathrm{mg} / \mathrm{kg})$ (Webster Veterinary). Eighteen-gauge gavage tubing (Fine Science Tool) was placed in the trachea, and $1 \times 10^{7}$ lymphangioleiomyomatosis cells labeled with Cy5.5 $(\sim 10 \mathrm{nmol})$ and ${ }^{99 \mathrm{~m}} \mathrm{Tc}$ ( $\left.\sim 30 \mathrm{MBq} ; 0.8 \mathrm{mCi}\right)$ in $150 \mu \mathrm{L}$ of PBS/ $\mathrm{MgCl}_{2}$ were administered intratracheally. After $15 \mathrm{~min}$, the mice underwent euthanasia, and a thoracotomy was performed to enucleate the lung tissues.

SPECT and CT images were acquired using a NanoSPECT/CT scanner (Bioscan, Inc.) equipped with $4 \mathrm{NaI}(\mathrm{Tl})$ detectors. For SPECT imaging, 9-pinhole apertures with a diameter of $1.4 \mathrm{~mm}$ were used on each detector. The energy window was set at $140 \mathrm{keV} \pm 20 \%$. SPECT imaging was performed at 24 projections per rotation and $300 \mathrm{~s}$ per projection. The projected data were reconstructed using HiSPECT software (Scivis) and a dedicated orderedsubsets expectation maximization algorithm using multiplexed multipinhole SPECT technology. Voxel size and gaussian filter size (full width at half maximum) were set at 0.3 and $1.9 \mathrm{~mm}$, respectively. The iterative update number (subset $\times$ iteration) was 27. CT was performed at $45 \mathrm{kVp}, 177 \mu \mathrm{A}$, and 240 projections per rotation using a complementary metal oxide semiconductor detector with a $96-\mu \mathrm{m}$ pixel size. Projected data were reconstructed by setting the voxel size to $100 \mu \mathrm{m}$.

After in vivo SPECT/CT imaging, the lung tissue was excised and imaged ex vivo using the same protocol. We then positioned the tissue under the FLARE imaging system (fluorescence-assisted resection and exploration; Curadel, LLC) for acquisition of 700-nm NIR fluorescence and color images, as described in detail previously $(12,13)$. After imaging the tissue, we placed the extracted lung tissues in $2 \%$ paraformaldehyde in PBS for 30 min before mounting them on the Tissue-Tek OCT (Sakura Finetek). The sample was frozen in liquid nitrogen and cryosectioned into $30-\mu \mathrm{m}$-thick sections for fluorescence imaging, autoradiography, and HE staining. A thickness of $30 \mu \mathrm{m}$ was chosen because the autoradiography signal was otherwise limiting. Three FMs were placed around the tissue. After autoradiography scanning and HE staining, anti-GFP antibody staining was performed using the optimized protocol as described above. The dual-channel NIR fluorescence and color images were obtained using a 55i fluorescence microscope (Nikon) equipped with an encoded motorized stage (Prior Scientific). To obtain the 700-nm fluorescence image, the xenon lamp was passed through a $650 / 45-\mathrm{nm}$ band-pass excitation filter, a 680-nm low-pass dichroic filter, and a 710/50-nm band-pass emission filter. For 800-nm fluorescence imaging, the xenon lamp was passed through a 750/50-nm band-pass excitation filter, a 810-nm low-pass dichroic filter, and an 824/47-nm band-pass emission filter. The dual NIR fluorescence and autoradiography images were then merged with the HE histology image using the 3 prepositioned FMs, and the weighted center of mass was calculated by Image J software, version $1.45 \mathrm{q}$. 


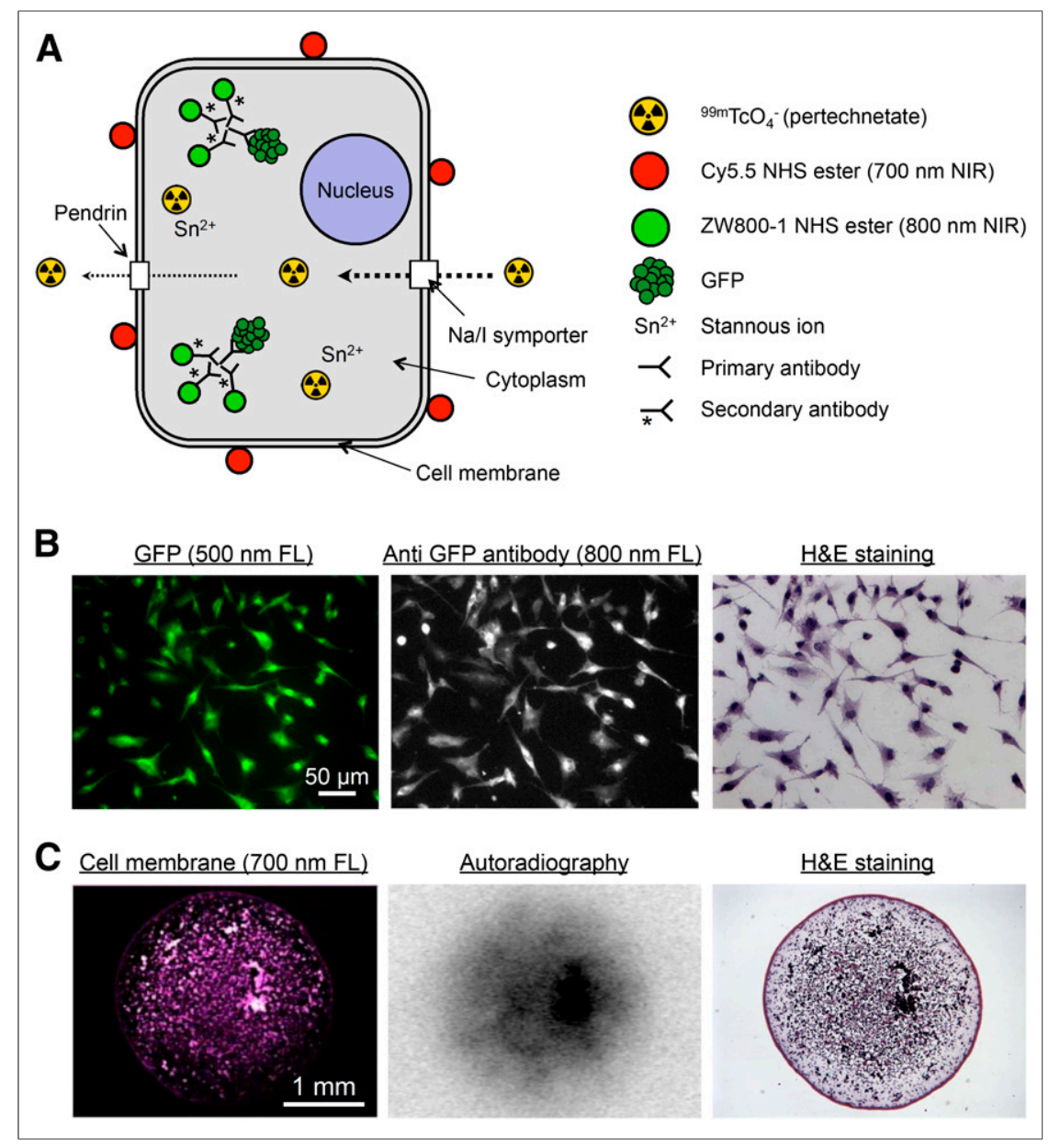

FIGURE 3. Multimodality labeling of single cells. (A) Summary of various NIR fluorescent and radioactive molecules used to label lymphangioleiomyomatosis cells. Cy5.5 NHS ester $(700-\mathrm{nm}$ NIR) was conjugated to cell membrane. ZW800-1 (800-nm NIR)-conjugated secondary antibody was used for immunofluorescence staining of GFP. ${ }^{99 m T c-p e r t a c h n e t a t e ~ w a s ~ a c c u m u l a t e d ~ v i a ~}$ sodium iodide symporter, and stannous ion $\left(\mathrm{Sn}^{2+}\right)$ was used to improve retention of radioisotope in cell. (B) Immunofluorescence staining of GFP. Shown is rabbit polyclonal anti-GFP antibody and ZW800-1 conjugated donkey antirabbit secondary antibody staining of lymphangioleiomyomatosis cells expressing GFP. The 500-nm fluorescence signal for GFP was imaged before HE staining, and 800-nm fluorescence and color microscopy was performed after HE staining. (C) Multimodality microscopy of lymphangioleiomyomatosis cells. Images are for 4-mm-diameter spot of lymphangioleiomyomatosis cells on glass slide. Note single-cell resolution by NIR fluorescence and $\mathrm{HE}$. FL = fluorescence.

\section{RESULTS}

\section{Optimization of the FM}

The chemical structures and optical spectra of HSA700 and HSA800 are shown in Figure 1. Since the half-life of ${ }^{99 m} \mathrm{Tc}$ is relatively short $(6 \mathrm{~h})$ and a stable chemical reaction is required, we used a simple and facile cross-linking reaction to conjugate albumin to the glass slide while simultaneously trapping ${ }^{99 \mathrm{~m}} \mathrm{Tc}-$ pertechnetate in the protein mesh. The type of slide most commonly used in clinical histopathology laboratories is the "plus" slide. The primary amines conjugated to the surface of the plus slide help tissue adhere and also serve as the matrix for FM conjugation.

Preparing FMs that were perfectly homogeneous, however,

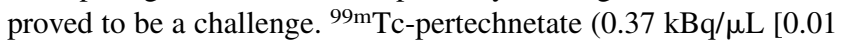
$\mu \mathrm{Ci} / \mu \mathrm{L}])$, unlabeled HSA $(100 \mu \mathrm{M})$, HSA700 $(1 \mu \mathrm{M})$, and HSA800 $(1 \mu \mathrm{M})$ were fixed in PBS, $\mathrm{pH} 7.4$, at several different concentrations of glutaraldehyde $(1 \%, 3 \%$, and $5 \%)$ and with dif- ferent concentrations of dimethyl sulfoxide $(0 \%, 5 \%, 10 \%$, and $15 \%)$ for $15 \mathrm{~min}$ at RT. The NIR fluorescence signal of HSA700 and HSA800 was imaged before and after HE staining to ensure that the FM was stable. Under all conditions tested, the signal intensity and shape of FMs were not altered significantly by HE staining, and the FM was visible by brightfield microscopy (Fig. 2). However, the homogeneity of the FMs was improved by increasing the concentrations of glutaraldehyde and dimethyl sulfoxide. Optimized conditions for FM production were found to be $100 \mu \mathrm{M}$ unlabeled HSA, $1 \mu \mathrm{M}$ HSA700, $1 \mu \mathrm{M}$ HSA800, $0.37 \mathrm{kBq}(0.01$ $\mu \mathrm{Ci})$ of ${ }^{99 \mathrm{~m}} \mathrm{Tc}$ pertechnetate per microliter, $3 \%$ glutaraldehyde, and $15 \%$ dimethyl sulfoxide in PBS ( $\mathrm{pH} \mathrm{7.4)} \mathrm{for} 15 \mathrm{~min}$ at RT.

\section{Cell Labeling and Imaging}

To create single cells that were NIRfluorescent at 700 and $800 \mathrm{~nm}$, as well as radioactive, lymphangioleiomyomatosis cells stably coexpressing sodium iodide symporter and GFP were incubated with ${ }^{99 m}$ Tc-pertechnetate and 700-nm Cy5.5 NHS (Fig. 3A). After fixation, GFP was detected using an unlabeled primary antibody and an 800-nm NIR fluorescent secondary antibody (Fig. 3B). Although expression of sodium iodide symporter alone is sufficient to accumulate ${ }^{99 m}$ Tc-pertechnetate in a cell $(14,15)$, we found that incubation with $\mathrm{Sn}^{2+}$, which diffuses across the cell membrane, accumulates intracellularly, and reduces $\mathrm{Tc}^{7+}$, helped to retain the radioisotope.

The inherent differences in sensitivity, specificity, and resolution among microscopic NIR fluorescence imaging, autoradiography, and HE staining are shown in Figure 3C. In particular, currently available phosphor imaging cassettes are not designed for high-energy isotopes, resulting in significant blurring of what would otherwise be single-cell resolution of the autoradiography signal.

\section{Microscopic Analysis of Macroscopic \\ Radioscintigraphic Images}

The 700-nm NIR fluorescent and radioactive lymphangioleiomyomatosis cells were administered intratracheally into CD-1 mice (Fig. 4A), and in vivo SPECT/CT imaging was performed (Fig. 4B). After surgical removal of the lung, SPECT/CT, color, and 700-nm fluorescence images were obtained (Fig. 4B) and a portion of lung tissue having both optical and nuclear signal was excised and processed for immunofluorescence using an 800-nm NIR fluorophore-conjugated antibody. As shown in Figure $4 \mathrm{C}$, it was possible to analyze 2 independent channels (700 and $800 \mathrm{~nm}$ ) of NIR fluorescence, as well as autoradiography, on the same specimen and on the same slide, with all datasets able to be coregistered using the FMs. Both NIR fluorescent and HE optical imaging could be performed with single-cell resolution (Fig. 4D). 


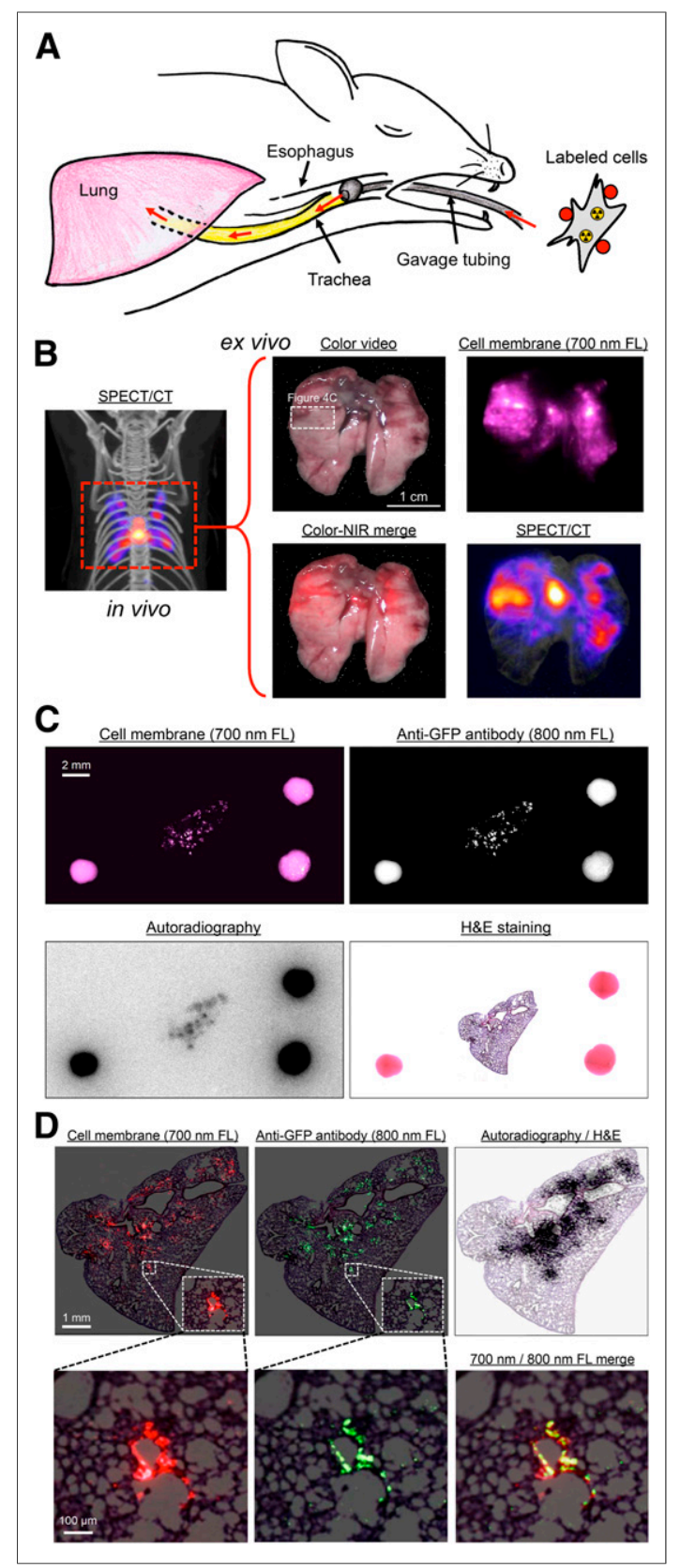

FIGURE 4. In vivo and ex vivo multifunctional imaging. (A) Schematic representation of in vivo mouse model. Labeled lymphangioleiomyomatosis cells (Fig. $3 \mathrm{~A}$ ) were administered intratracheally into $C D-1$ mice using gavage tubing. (B) In vivo maximal-intensity-projection SPECT/CT image (left) and ex vivo color, $700-\mathrm{nm}$ fluorescence, color-NIR merge (700-nm signal is pseudocolored in red), and maximal-intensityprojection SPECT/CT images (right) of excised lung. Images shown are representative of 3 independent experiments. White rectangle delineates excised tissue sample studied in C. (C) Same-slide 700-nm NIR fluorescence (cell membrane staining), 800-nm NIR fluorescence (anti-GFP staining), autoradiography, and HE staining of lymphangioleiomyomatosis cells in lung tissue of CD-1 mouse. The 2-mm-diameter multimodal FMs visible in each image were used to coregister datasets. (D) Same as C but at higher magnification, with 700-nm NIR fluorescent signal pseudocolored in red, 800-nm NIR fluorescent signal pseudocolored in green, and autoradiography signal superimposed on $\mathrm{HE}$ image. $\mathrm{FL}=$ fluorescence.
As might be expected on the basis of the experimental protocol, lymphangioleiomyomatosis cells were found to be distributed peribronchially (Fig. 4D).

\section{DISCUSSION}

This study addressed a fundamental problem in the field of nuclear medicine, the validation of macroscopic radioscintigraphic signals at the microscopic level. Previously, Axente et al. introduced coregistration between autoradiography and microscopic images (e.g., images of cell proliferation and HE) acquired from consecutive tissue sections using the deformable registration method (16). However, it has been a challenge to validate the radioscintigraphic signal and microscopic images on the same slide.

Having already optimized 2 channels of NIR fluorescence microscopy with HE staining on the same slide (Gibbs et al., unpublished data, September 2014), we assumed that adding the autoradiography dataset would be straightforward. On the contrary, coregistration of datasets between optical and nuclear imaging was found to be a major issue because autoradiography lacks identifiable landmarks and was acquired the night before the optical images. Even when a dual functional diagnostic agent (i.e., SPECT/NIR fluorescent probe) has been used for surgical navigation of resecting sentinel lymph nodes, a similar problem has been reported $(17,18)$. To circumvent this issue, we have described a simple, inexpensive FM that can be applied to a standard plus slide and permits rotation, translation, and morphing of datasets as needed among bright-field microscopy, NIR fluorescence immunomicroscopy, and autoradiography. This FM can be created with commercially available reagents in only $15 \mathrm{~min}$, which is an important point when considering the short half-life of many SPECT and PET radiotracers.

The biologic example we have shown in this study, namely the tracking of lymphangioleiomyomatosis cells after administration into the airspaces of the lung, actually has important implications. Our group recently described a new animal model of lymphangioleiomyomatosis and the related disease tuberous sclerosis complex that recapitulates the homing of lymphangioleiomyomatosis/ tuberous sclerosis complex cells to virtually every lymph node basin in the body (11). However, it is extremely difficult to study the mechanism of cellular translocation and lymph node homing because the process involves single cells. Armed with the technology described in this paper, it should now be possible to track cells using in vivo imaging methods and to analyze the source of measured signals at the microscopic level. This same strategy could be used to study similar processes, such as the early steps of cancer metastasis.

Nevertheless, our study also had limitations. Currently available phosphor imaging systems are not designed for high-energy $\gamma$ radioisotopes, and significant degradation of resolution occurs (e.g., Fig. 4C). Estimates of full-width-at-half-maximum resolution for ${ }^{99 \mathrm{~m}} \mathrm{Tc}$ and ${ }^{18} \mathrm{~F}$ are 350 and $460 \mu \mathrm{m}$, respectively (19). Fortunately, recent innovations in " $\gamma$ microscopes" and other highresolution techniques (20-22) suggest that a revolution in autoradiography is imminent, and our FMs will likely prove useful in validating this new technology. Improved resolution and quantitative information can also be achieved using a $\beta$ imager (e.g., the BetaImager [Biospace Lab]) by replacing ${ }^{99 \mathrm{~m}} \mathrm{Tc}$ in our example with a $\beta$-emitting radionuclide (e.g., ${ }^{3} \mathrm{H}$ and ${ }^{14} \mathrm{C}$ ), provided that its chemical form is trapped as well as ${ }^{99 \mathrm{~m}} \mathrm{Tc}$.

The ultimate use of the FMs we describe is to bridge the microscopic-macroscopic divide. As autoradiography technology 
evolves to a higher resolution, one can envision the day when a specimen is imaged in vivo using SPECT or PET and then resected and processed onto slides as we describe. NIR fluorescence could be used either to validate the source of the radioactive signal (using specific antibodies, for example) or to identify NIR fluorophore-labeled cells or tissues. Provided that the spacing and rotation between the sections are known, the high-resolution autoradiography images could then be assembled into a 3-dimensional dataset and coregistered with the in vivo image as has been shown previously (23). Someday, one might even have to blur the autoradiography dataset to qualitatively match the in vivo dataset. That day, in fact, would signal that the microscopicmacroscopic divide has finally been conquered.

\section{CONCLUSION}

We have developed a specialized FM and processing techniques that permit same-slide autoradiography, 700-nm and 800-nm NIR fluorescence, and HE histopathologic analysis at the single-cell level. FM marker preparation takes only 15 min using readily available reagents. Using this technology, macroscopic nuclear images can be validated at the microscopic level.

\section{DISCLOSURE}

The costs of publication of this article were defrayed in part by the payment of page charges. Therefore, and solely to indicate this fact, this article is hereby marked "advertisement" in accordance with 18 USC section 1734. Dr. Frangioni is currently CEO of Curadel, LLC, a for-profit company that has licensed FLARE technology from Beth Israel Deaconess Medical Center. This study was supported by grants from the National Institutes of Health (NCI grant R01-CA134493, NIBIB grant R01-EB-010022, and NIBIB grant R01-EB011523 ) and by a grant from the Tuberous Sclerosis Alliance. The views expressed are solely those of the authors and do not necessarily reflect those of the National Institutes of Health. No other potential conflict of interest relevant to this article was reported.

\section{ACKNOWLEDGMENTS}

We thank David Burrington, Jr., for editing and Eugenia Trabucchi for administrative assistance.

\section{REFERENCES}

1. Patil V, Gada K, Panwar R, et al. Imaging small human prostate cancer xenografts after pretargeting with bispecific bombesin-antibody complexes and targeting with high specific radioactivity labeled polymer-drug conjugates. Eur $J$ Nucl Med Mol Imaging. 2012;39:824-839.

2. Wuest M, Trayner BJ, Grant TN, et al. Radiopharmacological evaluation of 6deoxy-6- $\left[{ }^{18} \mathrm{~F}\right]$ fluoro-D-fructose as a radiotracer for PET imaging of GLUT5 in breast cancer. Nucl Med Biol. 2011;38:461-475.
3. Abasolo I, Pujal J, Rabanal RM, et al. FDG PET imaging of Ela1-myc mice reveals major biological differences between pancreatic acinar and ductal tumours. Eur J Nucl Med Mol Imaging. 2009;36:1156-1166.

4. Müller C, Forrer F, Schibli R, Krenning EP, de Jong M. SPECT study of folate receptor-positive malignant and normal tissues in mice using a novel ${ }^{99 \mathrm{~m}} \mathrm{Tc}$ radiofolate. J Nucl Med. 2008;49:310-317.

5. Oka S, Hattori R, Kurosaki F, et al. A preliminary study of anti-1-amino-3- ${ }^{18} \mathrm{~F}-$ fluorocyclobutyl-1-carboxylic acid for the detection of prostate cancer. $\mathrm{J} \mathrm{Nucl}$ Med. 2007;48:46-55.

6. Ankle MR, Joshi PS. A study to evaluate the efficacy of xylene-free hematoxylin and eosin staining procedure as compared to the conventional hematoxylin and eosin staining: an experimental study. J Oral Maxillofac Pathol. 2011;15:161-167.

7. De Saint-Hubert M, Prinsen K, Mortelmans L, Verbruggen A, Mottaghy FM. Molecular imaging of cell death. Methods. 2009;48:178-187.

8. Valencia JC, Pacheco-Rodriguez G, Carmona AK, et al. Tissue-specific reninangiotensin system in pulmonary lymphangioleiomyomatosis. Am J Respir Cell Mol Biol. 2006;35:40-47.

9. Choi HS, Nasr K, Alyabyev S, et al. Synthesis and in vivo fate of zwitterionic near-infrared fluorophores. Angew Chem Int Ed Engl. 2011;50:6258-6263.

10. Hyun H, Bordo MW, Nasr K, et al. cGMP-compatible preparative scale synthesis of near-infrared fluorophores. Contrast Media Mol Imaging. 2012;7:516-524.

11. Liu F, Lunsford EP, Tong J, et al. Real-time monitoring of tumorigenesis, dissemination, \& drug response in a preclinical model of lymphangioleiomyomatosis/tuberous sclerosis complex. PLOS ONE. 2012;7:e38589.

12. Tanaka E, Choi HS, Fujii H, Bawendi MG, Frangioni JV. Image-guided oncologic surgery using invisible light: completed pre-clinical development for sentinel lymph node mapping. Ann Surg Oncol. 2006;13:1671-1681.

13. Troyan SL, Kianzad V, Gibbs-Strauss SL, et al. The FLARE intraoperative nearinfrared fluorescence imaging system: a first-in-human clinical trial in breast cancer sentinel lymph node mapping. Ann Surg Oncol. 2009;16:2943-2952.

14. Bengel FM, Schachinger V, Dimmeler S. Cell-based therapies and imaging in cardiology. Eur J Nucl Med Mol Imaging. 2005;32(suppl 2):S404-S416.

15. Seo JH, Jeon YH, Lee YJ, et al. Trafficking macrophage migration using reporter gene imaging with human sodium iodide symporter in animal models of inflammation. J Nucl Med. 2010;51:1637-1643.

16. Axente M, He J, Bass CP, et al. Comprehensive approach to coregistration of autoradiography and microscopy images acquired from a set of sequential tissue sections. J Nucl Med. 2011;52:1621-1629.

17. Zhang R, Lu W, Wen X, et al. Annexin A5-conjugated polymeric micelles for dual SPECT and optical detection of apoptosis. J Nucl Med. 2011;52:958-964.

18. Buckle T, van Leeuwen AC, Chin PT, et al. A self-assembled multimodal complex for combined pre- and intraoperative imaging of the sentinel lymph node. Nanotechnology. 2010;21:355101.

19. Knol RJ, de Bruin K, de Jong J, van Eck-Smit BL, Booij J. In vitro and ex vivo storage phosphor imaging of short-living radioisotopes. J Neurosci Methods. 2008; 168:341-357.

20. Miller BW, Barrett HH, Furenlid LR, Barber HB, Hunter RJ. Recent advances in BazookaSPECT: real-time data processing and the development of a gamma-ray microscope. Nucl Instrum Methods Phys Res A. 2008;591:272-275.

21. Chen L, Gobar LS, Knowles NG, Liu Z, Gmitro AF, Barrett HH. Direct imaging of radionuclide-produced electrons and positrons with an ultrathin phosphor. $J$ Nucl Med. 2008;49:1141-1145.

22. Chen L, Gobar LS, Knowles NG, Wilson DW, Barrett HH. Direct chargedparticle imaging system using an ultra-thin phosphor: physical characterization and dynamic applications. IEEE Trans Nucl Sci. 2009;56:2628-2635.

23. Humm JL, Ballon D, Hu YC, et al. A stereotactic method for the three-dimensional registration of multi-modality biologic images in animals: NMR, PET, histology, and autoradiography. Med Phys. 2003;30:2303-2314. 